\title{
Performance Characterization and Modeling of Serverless and HPC Streaming Applications
}

\author{
Andre Luckow ${ }^{1,2,3,4}$, Shantenu Jha ${ }^{1,5}$ \\ ${ }^{1}$ RADICAL, ECE, Rutgers University, Piscataway,NJ 08854, USA \\ ${ }^{2}$ BMW Group, Munich, Germany \\ ${ }^{4}$ Ludwig Maximilian University, Munich, Germany \\ ${ }^{3}$ Clemson University, Clemson, SC 29634, USA \\ ${ }^{5}$ Brookhaven National Laboratory, Upton, NY, USA
}

\begin{abstract}
Experiment-in-the-Loop Computing (EILC) requires support for numerous types of processing and the management of heterogeneous infrastructure over a dynamic range of scales: from the edge to the cloud and HPC, and intermediate resources. Serverless is an emerging service that combines high-level middleware services, such as distributed execution engines for managing tasks, with low-level infrastructure. It offers the potential of usability and scalability, but adds to the complexity of managing heterogeneous and dynamic resources. In response, we extend Pilot-Streaming to support serverless platforms. Pilot-Streaming provides a unified abstraction for resource management for HPC, cloud, and serverless, and allocates resource containers independent of the application workload removing the need to write resource-specific code. Understanding of the performance and scaling characteristics of streaming applications and infrastructure presents another challenge for EILC. StreamInsight provides insight into the performance of streaming applications and infrastructure, their selection, configuration and scaling behavior. Underlying StreamInsight is the universal scalability law, which permits the accurate quantification of scalability properties of streaming applications. Using experiments on HPC and AWS Lambda, we demonstrate that StreamInsight provides an accurate model for a variety of application characteristics, e.g., machine learning model sizes and resource configurations.
\end{abstract}

Index Terms-Serverless, Streaming, Performance, HPC

\section{INTRODUCTION}

The integration of experimental instruments, the Internetof-Things (IoT), compute and data infrastructure - from the edge to HPC and cloud - is increasingly important to enable new scientific discoveries and advances [1]. Experiment-inthe-Loop Computing (EILC) describes a new type of application and infrastructure, where experimental capabilities are augmented with compute and data capabilities to enable more intelligent experiments. Stream processing capabilities are essential for EILC to enable realtime insights on data feeds from myriad sources [2]. Applications for stream processing range from connected and autonomous vehicles [3], realtime analysis of astronomy data to other scientific experiments, such as light sources [4]. For example, Synchrotron light source experiments, such as those at the National Synchrotron Light Sources II (NSLS-II) [5] or the X-Ray Free Electron Laser (XFEL) light sources. EILC and stream processing can be used for detecting events of interests, pre-processing data, and steering of simulations and instruments.

EILC require infrastructure - edge, cloud, and HPC, which are complementary to each other: edge is suited for realtime processing close to the data source, HPC is better suited for high-end computational and data-intensive tasks, such as simulations. Cloud platforms provide commoditized capabilities for streaming and data analytics.

A new, emerging platform is serverless — which abstract away most, but not all resource management concerns, such as the allocation of nodes, containers and the management of the processing framework [6], [7]. Serverless is well suited for data-parallel tasks, including streaming.

EILC applications need to be able to orchestrate streaming workloads comprising of dependent and parallel tasks. Managing such workloads on heterogeneous infrastructure from HPC to serverless is associated with several challenges:

Development \& Deployment: There are a lack of abstractions for efficiently managing task-based workloads on serverless, such as workloads requiring complex inter-task dependencies and parallelism [8]. As a result, application codes need to be carefully and manually partitioned and wrapped into serverless APIs. While serverless promises to reduce the need for manual resource management, it still requires applications to configure and adjust resource-related parameters, e. g., the concurrency and memory per container.

Interoperability: Abstractions for resource and task management differ between HPC and serverless; HPC abstractions are low-level and infrastructure-centric compared to serverless abstractions. Additionally, differences between platform providers exist. This lack of interoperability complicates the use of multiple platforms within an application.

Execution: Multiple layers of infrastructure, middleware, and execution engines make it difficult to monitor, understand and predict the performance of an application. For serverless, many details of the runtime are abstracted and opaque further complicating the performance understanding. The determination of appropriate performance metrics and the manual evaluation of streaming systems under different workloads and resource configurations is a complex undertaking.

To overcome the development, interoperability, and execution challenges, we extended Pilot-Streaming to serverless platforms. Pilot-Streaming is a unifying abstraction based on the pilot abstraction [9] for resource management across heterogeneous platforms. The pilot abstraction decouples resource allocation from workload execution, enabling scalable and flexible execution of tasks. Tasks are self-contained sets of operations that possess different characteristics, e.g., related 
to its granularity, compute, memory and I/O demands. Dataparallel and streaming workloads are typically comprised of many short-running tasks which in the case of streaming are generated in response to incoming data. With the extension to serverless, Pilot-Streaming provides a unified way to acquire HPC, clouds and serverless resources enabling developers to compose tasks and express task-level parallelism efficiently.

We developed the StreamInsight framework for analyzing and predicting the performance of streaming systems and applications. It is based on the Universal Scalability Law (USL) [10], which captures the underlying properties of the system that limit scalability. StreamInsight can enhance the experimental design, automation and analysis of streaming systems. We demonstrate its capabilities using a machine learning streaming application to detecting abnormal behavior on different infrastructures, in particular on serverless using Kinesis [11] as a message broker and AWS Lambda [12] for processing, and on HPC machines using Kafka [13] as broker and Dask [14] for processing.

This paper is structured as follows: In section II we provide essential background and related work. We continue with a discussion of Pilot-Streaming on serverless in section III. In section IV, we present the theoretical foundation and architecture of StreamInsight. Further, we validate the system by performing different experiments on different infrastructures and application characteristics. We conclude with a discussion of the results and future work in section V.

\section{BACKGROUND AND RELATED WORK}

In this section, we present important background and related work on serverless (section II-A), and benchmarking and performance modeling of streaming applications (section II-B).

\section{A. Serverless}

The term serverless [7] is most commonly used for Function-as-a-Service (FaaS) platforms, such as AWS Lambda [12], Google Cloud Functions and Azure Functions. It is a computing paradigm that allows the scalable and fault-tolerant execution of functions in response to defined events without the need to consider low-level concerns, such as resource management, provisioning and scaling with respect to the number of events. A serverless function comprises of a self-contained piece of code that implements an interface defined by the platform. It is then deployed on the FaaS infrastructure, which automatically instantiates, executes, scales and monitors the function.

Serverless tightly integrates the abstraction for expressing computational tasks with its instantiation in the runtime system. The function code is required to be stateless, i.e., the state needs to be managed and stored outside the function. Further, the function is subject to strict constraints, e.g., the container size is limited to a single core, a defined amount of memory, and the runtime is subject to a walltime (currently 15 minutes processing time per event for Lambda). The cloud platform provides a high-level SLA concerning the available compute and data resources. Serverless is increasingly relevant for scientific applications, especially in conjunction with HPC capabilities [15], [16], [17]. The event-driven nature of streaming applications makes them ideally suited to utilize the serverless paradigm.

\section{B. Streaming Performance and Modeling}

While there is comprehensive work on benchmarking streaming systems [18], [19], typically, these benchmarks do not reflect the complex and highly dynamic requirements of real-world scientific streaming applications. Fox et al. propose the concept of Big Data Ogres to describe well-understood application characteristics, which served as a basis for the development of a set of benchmarks that encapsulate commonalities of these applications [20]. The concepts of Mini-Apps was introduced in the domain of data-intensive HPC apps by Sukumar et al. [21]. Luckow et al. refined this concept for streaming apps [4].

Increasingly statistical methods are used to understand performance and to make predictions, e.g., for resource (re)configurations decisions [22]. Kremer-Herman et al. [23] propose a model for recommending the optimal infrastructure configuration for master/worker applications. Ernest [24] is a system that combines analytical modeling and data derived from a small set of performance counters and sample runs. Cherrypick [25] uses a black-box model based on Bayesian optimization to find optimal resource configurations for Big data frameworks in the cloud. Caladrius [26] is a streaming forecasting system und provides a predictive model for traffic and an analytical model for the processing system.

The main limitations of these approaches are that they only investigate a small part of the stream processing pipeline and are often constrained to specific frameworks. The experimental validation is often done using simple examples that are not representative of real-world scientific applications. For example, Caladrius is solely evaluated using a wordcount example and parallelism of 8 .

\section{Pilot-Streaming: Extension to Serverless}

In this section, we discuss the extension of Pilot-Streaming to support serverless platforms. Pilot-Streaming [4] provides a unified interface for resource management on serverless and HPC, and supports the development, deployment, and execution of streaming applications. Specifically, it removes the need for applications to use platform-specific serverless API, which commonly differs between cloud platforms. Further, it provides a unified way to express task-based workloads, to monitor the execution of tasks and to handle faults.

Underlying Pilot-Streaming is the pilot abstraction, which provides a unified platform- and infrastructure-agnostic way to acquire resources and execute tasks on them. We extended Pilot-Streaming to support the allocation of serverless resource containers. After the acquisition of the resources, applications can manage their workload typically comprised of parallel and dependent tasks on these resources [9], [27]. The tools support workloads comprising of different sets of dependent and 


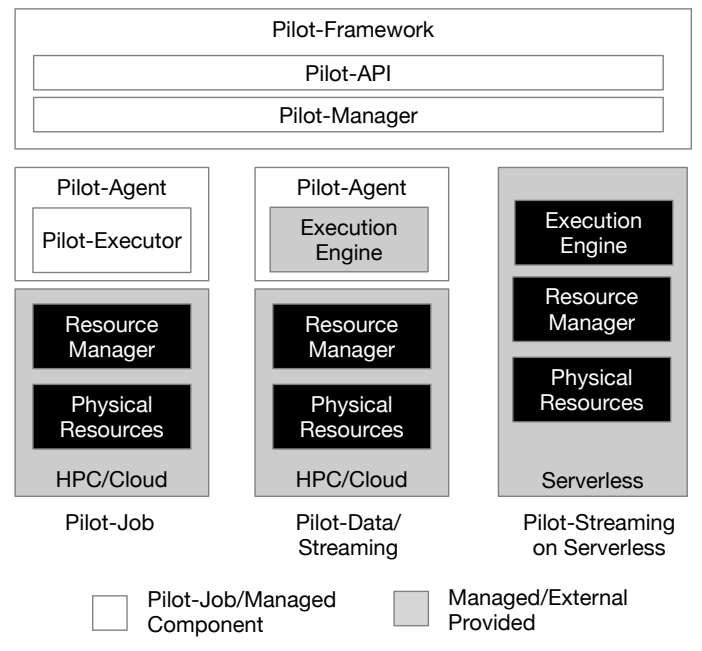

Fig. 1: Pilot Abstraction on Heterogeneous Platforms: HPC, Cloud, and Serverless: The pilot abstraction can integrate with platforms and infrastructures at various levels. Pilot-Streaming on serverless abstracts the serverless-specific resource acquisition enabling a unified way to manage tasks on HPC, clouds, and serverless.

parallel tasks with different characteristics, such as task granularity, compute, memory, and I/O demands. Data-parallel and streaming workloads typically comprise of many short-running tasks that in the case of streaming are generated in response to incoming data.

The pilot abstraction is exposed via the Pilot-API and consists of two entities: pilot-job which represents a user-defined set of resources, and compute-unit which is a task representing a self-contained set of operations and is the key abstraction for expressing the application workload. Resources can be requested using the pilot-job class. Once the pilot-job has been instantiated, compute-units can be submitted to this instance.

Figure 1 illustrates the different types of infrastructures supported by the pilot abstraction. Initially, pilot-jobs [9] focused on the provisioning and management of HPC and cloud resources (compute nodes, VMs), and the execution of the application workload on these. Pilot-Data [28] and Pilot-Streaming [4] extended the pilot abstraction to externally provided distributed execution engines, e.g., Spark [29] and Dask [14]. This extension allowed the support of higher-level, data-intensive workloads that rely on the capabilities of these execution engines. With serverless the operational responsibility for the execution engine shifts from the application to the infrastructure provider.

Pilot-Streaming is based on a modular architecture and provides several plugins for supporting a diverse set of infrastructures and frameworks (see Figure 2). The HPC and cloud plugins [4] enable the usage of the pilot abstraction for streaming applications on these platforms, i.e., it supports the deployment and execution of Kafka and Dask on HPC and EC2 cloud instances. Using the plugin architecture, Pilot-Streaming was extended to support the serverless services AWS Lambda and Kinesis. The Pilot-Manager continues to provide a unified interface - the Pilot-API - for running compute-units on these platforms, but also serves as an orchestrator for managing data

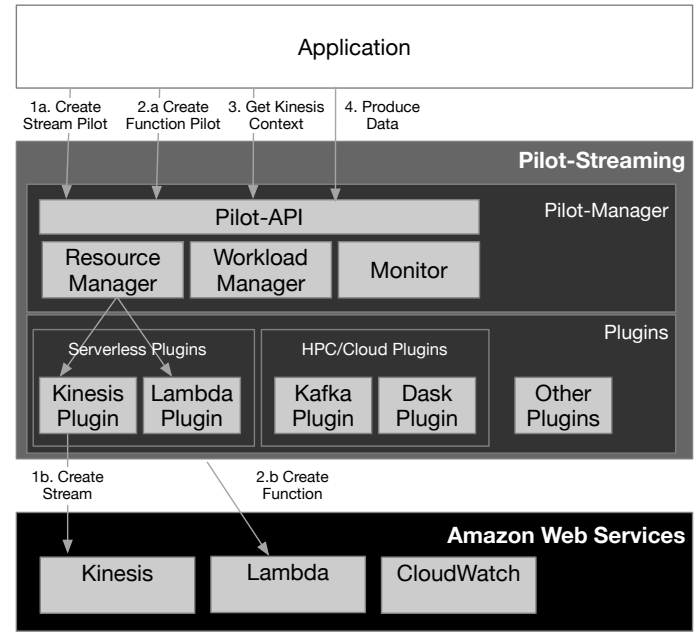

Fig. 2: Pilot-Streaming on Serverless High-Level Architecture and Interactions: Pilot-Streaming enables the unified management of batch and streaming compute tasks on serverless infrastructure. It orchestrates the setup of Kinesis and Lambda and manages the streaming workload.

and compute across the different platforms.

In the following, we discuss the interactions between the components of Pilot-Streaming. First, the application typically initiates a Kinesis Pilot and allocates a stream with a defined number of shards (see step 1a and $b$ in Figure 2). For this purpose, the user needs to create a Pilot-Description, which provides a normative way to specify resources for a streaming broker, e. g., the number of topic shards for Kinesis and Kafka can be specified using the same attribute. Pilot-Streaming then allocates resources for Kinesis using the platform-specific plugin, which encapsulates the necessary details. In cases where the message broker is outside of the managerial scope of the application, this step can be skipped and the producing, and the consuming application can directly connect to the broker.

In step 2a and $b$, the Function Pilot for managing Lambda is created. Pilot-Streaming supports the execution of arbitrary Python code as Lambda function. Pilot-Streaming deploys the function on the serverless environment and automatically handle serialization of the function and its dependencies and the transfer to Lambda. Also, the usage of Lambda Layers is supported. The Pilot-Description is used to specify and control the parallelism in an infrastructure-agnostic way, while allowing the support for infrastructure-specific capabilities, such as layers or memory limits on Lambda.

Pilot-Streaming supports two usage modes: (i) the submission of arbitrary compute tasks to Lambda; (ii) the invoking of compute tasks in response to incoming data events. For the first usage mode, the pilot abstraction can be used to formulate common task-parallel patterns, e.g., for implementing data parallelism or compose complex DAGs. For the second usage mode, the Pilot-Description can be used to connect input data streams to the Lambda function. Then, the framework will automatically setup this mapping. A task is then automatically spawned in response to an event, e.g., a new message arrived at the broker. 
In summary, Pilot-Streaming simplifies the development, deployment, and execution of serverless streaming applications. In addition to providing a consistent way to allocate resources, it provides many functional enhancements, such as the ability to express task-level parallelism more efficiently than native FaaS APIs. The pilot abstraction provides a welldesigned and easy-to-use API for task-based workloads that can be utilized to express different types of parallelism, from bag-of-tasks, data-parallelism to streaming on heterogeneous infrastructure, making it particularly well-suited for workloads required to run on a diverse set of infrastructure. These capabilities provide the basis for achieving high-performance and scalable applications, which we discuss in the next section.

\section{Streaminsight: PERformanCE CHARACTERIZATION AND MODELING}

Understanding the complex and dynamically varying performance characteristics of streaming systems is necessary to ensure stable system execution by allocating sufficient resources and configuring frameworks appropriately. The performance depends on multiple variables, as the degree of parallelism and the available memory. The number of attributes in the involved systems is high, and often these attributes are not independent. Thus, performance data is challenging to collect, normalize, analyze, and compare. Furthermore, the combinatorial space of parameters is ample, and thus, a careful selection of the most significant factors to investigate is critical.

StreamInsight supports the end-to-end process of performance experimentation and modeling of streaming applications and systems from experimental design, implementation, automation to analysis and modeling. It can be used to identify and quantify bottlenecks, test streaming systems under various traffic loads, fine-tune system configurations, optimize resource allocations, and predict the performance of applications. Further, the system can serve as a building block for higher-level functionality, such as predictive autoscaling.

StreamInsight comprises of two components: the Streaming Mini-App framework [4] that simplifies data collection and automation of experiment, and a Universal Scalability Model (USL) [10] based performance modeling, analysis and prediction framework. USL provides a well-established analytical model as a foundation for understanding, explaining and predicting streaming systems. The Streaming Mini-App framework [4] is used to simulate complex streaming applications from data production, brokering to processing. The capabilities of the Streaming Mini-App framework allow application developers to automate the exploration of large parameter spaces of different frameworks and application configurations.

The Mini-App framework is built on Pilot-Streaming, which is used to manage resources, stream processing runtimes, and the message broker. We extended the Mini-App framework to support the collection of comprehensive performance data on serverless infrastructure in addition to HPC infrastructure and provide the ability to trace data across all components, i.e., data source, brokering and processing system. For this purpose, the framework assigns a unique run id, which is prop- agated to all involved components. This way events can be attributed to a specific benchmark runs.

The instrumentation system is architected in a modular way allowing the developer to easily add/remove metrics for all components. Currently, data can be collected from PilotStreaming, the message brokers, processing frameworks and cloud-based log services, such as AWS CloudWatch. The framework is architected in a modular way, i.e., the data collector can easily be extended to support new systems and infrastructure. It can simulate different data rates and characteristics (e.g., message sizes) aiding the collection of performance data. To conduct measurements at the maximum sustained throughput, the framework utilizes an intelligent backoff strategy during data production.

\section{A. Performance Model}

We build a model for understanding and predicting the throughput of a streaming system. We begin with a definition of the relevant variables. A streaming system consists of the message broker $(b r)$, and the processing system $(p x)$. The performance is measured by two metrics: the latency $(L)$ and throughput $(T) . L$ is defined as the time difference between two states, e.g., $L^{p x}$ is the time between arrival and processing of message in the processing system. $L^{b r}$ is the time between message production and its availability at the broker.

Throughput $T$ is defined as the number of events (e.g., messages) a system handles in a certain amount of time. The throughput of the stream processing system $\left(T^{p x}\right)$ depends upon the parallelism and the number of partitions $N^{p x}(p)$ and nodes $N^{p x}(n)$. We measure and analyze the maximum sustained throughput, i.e., the optimal load a streaming system can handle without performance deterioration (e.g., due to back-pressure) [30]. Further control parameters are the machine $M$, the message size $M S$, and the workload complexity $W C$. Table I summarizes the parameters of the model.

According to USL, $T(N)$ of a system is represented as follows:

$$
T(N)=\frac{N}{1+\sigma(N-1)+\kappa N(N-1)}
$$

For stream processing systems, $T=T^{p x}$ and $N=N^{p x}(p)$. The expression has two parameters: $\sigma$, which measures the serial overhead in the system, and $\kappa$, which captures the coherence between all processors. For example, $\sigma$ can be used to quantify overheads, such as serialization; $\kappa$ measures the all-to-all communication typically required, e.g., for sharing model parameters across all tasks. A $\sigma$ and $\kappa$ of 0 indicate optimal scalability. The larger both terms are, the worse the scalability. USL is well suited for modeling streaming systems and provides the right level of granularity while maintaining a high degree of interpretability. Further, USL does not require low-level timing to model the system. Gunther [31] showed that USL generalizes Amdahl's laws [32] and adds meaningful extensions, e.g., to explain performance degradations.

The different components of the physical system have a different impact on the model parameters. Due to the higher parallelism, larger $N^{p x}(p)$ and $N^{p x}(n)$ create more contention 


\begin{tabular}{|l|l|}
\hline$L$ & Overall Latency \\
\hline$L^{p x}$ & Latency Processing System \\
\hline$L^{b r}$ & Latency Broker System \\
\hline$T$ & Overall Throughput \\
\hline$T^{p x}$ & Throughput Processing System \\
\hline$T^{b r}$ & Throughput Broker System \\
\hline \hline$N^{p x}(n)$ & Number Nodes Processing System \\
\hline$N^{p x}(p)$ & Number Partitions Processing System \\
\hline$N^{b r}(n)$ & Number Nodes Broker System \\
\hline$N^{b r}(p)$ & Number Partitions Broker System \\
\hline \hline$M$ & Machine and Infrastructure \\
\hline$W C$ & Workload Complexity \\
\hline$M S$ & Message Size \\
\hline
\end{tabular}

TABLE I: Model Dependent, Independent and Control Variables

on shared resources, such as shared filesystems and networks. The ratio of $N^{p x}(p)$ to $N^{p x}(n)$ influences both $\sigma$ and $\kappa$ as it determines the amount of network traffic required to communicate between the partitions.

We assume a stable system operating at its maximum sustained throughput $T$, which is ensured by using an intelligent backoff strategy during data production. Further, overheads for initialization of the streaming application and infrastructures, i.e., the time for starting the streaming framework via the Pilot-Streaming, are not considered. We fit the data to USL using a non-linear regression model provided by the USL R package [33].

\section{B. Experiments and Characterization}

We characterize the performance of different infrastructures $(M)$ : (i) Kinesis/Lambda serverless processing on AWS, and (ii) Kafka/Dask stream processing on Wrangler and Stampede2 (both HPC). In both scenarios, we deploy a Streaming Mini-App framework, i.e., the synthetic data generator, the broker, and distributed execution engine on HPC, respectively AWS. For HPC, we use the XSEDE machines Stampede 2 and Wrangler. Each Wrangler node provides 48 cores and $128 \mathrm{~GB}$ RAM. On Stampede2, the Knights Landing nodes with 68 cores, 96 GB RAM were used. For serverless, we use AWS Lambda and Kinesis with different container sizes and numbers of partitions. For the synthetic data generator, we utilize m5. 4xlarge nodes with 16 cores and 64 GB memory.

We investigate the clustering algorithm K-Means [34] as a representative workload. K-Means is well understood and commonly used in streaming applications to detect abnormal behavior. K-Means has a complexity of $O(n c)$ with $n$ being the number of points and $c$ the number of cluster centroids. The algorithm comprises of two phases: First, K-Means computes the Euclidean distance between all points $(n)$ and the centroids $(c)$, which corresponds to a complexity of $O(n c)$. Then, the new centroid positions are computed by averaging the positions of all points assigned to a centroid.

We use the MiniBatch K-Means of scikit-learn [35] and update the K-Means model continuously based on the incoming data. The model is shared across tasks using file storage (S3 on AWS, Lustre filesystem on HPC). We vary the workload complexity $W C$ by using different numbers of centroids (between 128 and 8,192): the higher the number of centroids the more compute is necessary, and the $\mathrm{I} / \mathrm{O}$ for writing/reading

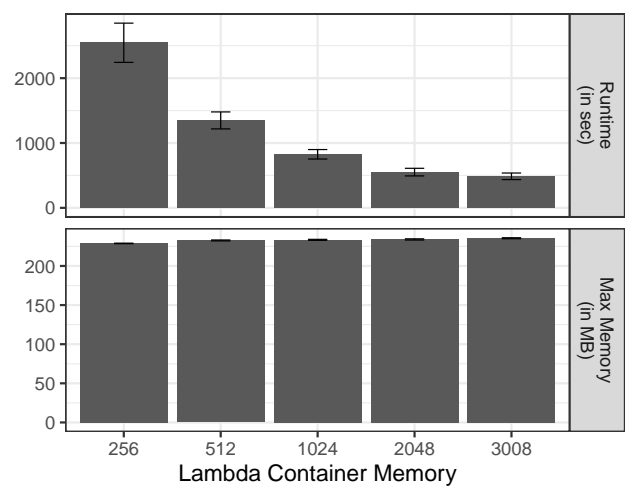

Fig. 3: Lambda Container Memory for 8,000 points and 1,024 centroids: Lambda containers with a larger amount of memory provide more compute capacity and thus, enable shorter runtimes. The fluctuation in the data is significantly lower for larger container sizes.

the model is increased. Further, we evaluate different message sizes $M S$ : $296 \mathrm{~kb}$ for 8,000 points, $592 \mathrm{~kb}$ for 16,000 points and $962 \mathrm{~kb}$ for 26,000 points.

For this experiment, we constrain the variables as follows: To meet the memory requirements of the application, we select an appropriate core/node ratio: $N^{p x}(n) / N^{p x}(p)$. On both Wrangler and Stampede2, we use 12 cores/nodes, which corresponds to $11 \mathrm{~GB}$ per core on Wrangler and $8 \mathrm{~GB}$ per core on Stampede2. Further, we use the same number of brokers and processing nodes: $N^{p x}(n)=N^{b r}(n)$.

1) Lambda Memory: An important parameter for computeunits executed within Lambda is the amount of memory. Figure 3 illustrates the runtime of the K-Means Lambda function broken down by the requested lambda container memory. We investigate the memory usage for a message size of $8 \mathrm{~K}$ points and 1024 clusters. Even though the actually used memory for the lambda function nearly remains constant, the runtime decreases with larger memory runtimes (currently max 3,008 $\mathrm{MB}$ ) indicating that AWS scales the CPU allotment proportional to the memory.

2) Lambda vs. Dask: Figure 4 investigates the processing latency per message $L^{p x}$ for different numbers of messages and centroids. For Lambda, we use 3 GB RAM containers. The processing latencies increase with the number of points and centroids for both Dask and Lambda due to the increase of the computational, I/O and memory demands of the processing function. For Lambda, the processing times remain stable with higher parallelisms, i. e., higher partition counts. The number of Lambda containers is managed completely by AWS. AWS never starts more containers than Kinesis partitions. During all experiments, we observed at most 30 concurrent containers.

While Kinesis/Lambda stream processing provides a predictable performance, for Dask $L^{p x}$ increases with the number of partitions indicating system contentions. This also results in a degradation of the throughput for larger $N^{p x}(p)$ as shown in Figure 5. For the more compute-intensive scenarios, i.e. in particular larger model sizes such as 8,192 clusters, a small speedup of up to 1.2 is observable for Dask/Kafka until 4 par- 


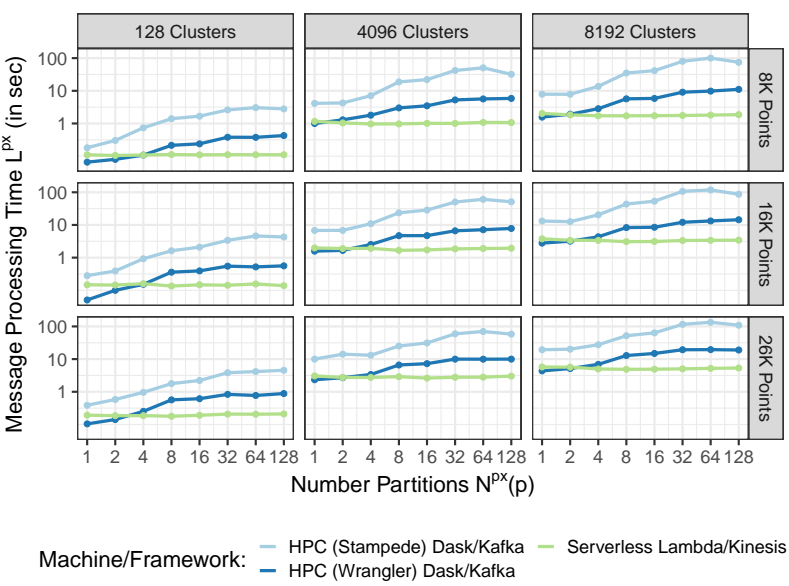

Fig. 4: Message Processing Time $L^{p x}$ for K-Means on AWS Lambda and HPC: Broken down by number partitions, message size, and workload complexity. The processing times increase with the message size and a higher number of clusters. While for Lambda the processing times remain constant with increasing parallelism, we observe a negative impact for Dask/Kafka on HPC due to the use of shared filesystem and network resources.

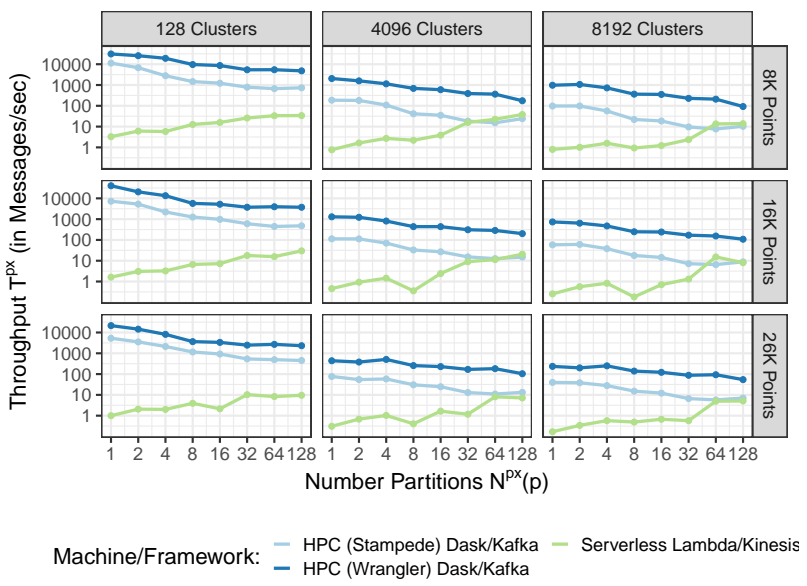

Fig. 5: Lambda and Dask Throughput $T^{p x}$ for K-Means on AWS Lambda and HPC: The increased processing times also impact the throughput and speedup. For scenarios with higher compute to $\mathrm{I} / \mathrm{O}$ ratio a small speedup is observable for Dask until 4 partitions.

titions. In the following, we analyze the data using USL.

\section{Model Analysis}

Figure 6 shows the throughput and the computed USL model for the different variables $M, W C$, and $M S$. The KMeans application is loosely coupled; all tasks process a partition independently with a minor synchronization for model updates. Further, there are shared I/O resources used by the broker and the processing engine in particular on HPC. These characteristics are reflected in the $\sigma$ and $\kappa$ coefficients of the USL model. $\sigma$ quantifies the serial overhead, while $\kappa$ measures the all-to-all communication between all nodes. All-toall communication can e.g. be required to update the model parameters between all tasks. We also noticed on both HPC environments severe performance degradation for larger $N$ due to I/O issues caused by the shared filesystem.

The scalability depends significantly on the infrastructure. While HPC provides a better absolute performance, cloud infrastructures are more predictable. For HPC the peak performance is already reached in many cases using a single partition. The system performance degrades with increased parallelisms due to contention and coherencies overheads. Running both data production, brokering, and processing (including complex coordination for sharing model parameters) on the shared filesystem is the likely cause. Lambda and Kinesis provide better resource isolation.

For Lambda, the throughput increases with the number of partitions thanks to the greater parallelism. Smaller message and model sizes have a higher throughput. USL produces a contention coefficient $\sigma$ and a coherence coefficient $\kappa$ of close to zero explaining almost optimal scalability. These small coefficients shows that Lambda containers are well isolated, providing a predictable performance enabling AWS to provide predictable SLAs.

For Dask, a $\sigma$ between 0.6 and 1 explains that the scalability bottleneck is caused by resource contentions, e.g., through shared memory, network and filesystem. However, $\kappa$ indicates that some significant coherency due to cross-communication between all processors, e. g., the synchronization of the model updates via the shared filesystem. Thus, the peak scalability of the system is already reached with a single partition. Further, it must be noted that Dask/Kafka was carefully fine-tuned to the HPC machine by carefully choosing the right memory/core ratio and the location of the Kafka data log files. However, the number of shared resources that impact performance is significantly larger on HPC than on serverless.

\section{Model Evaluation}

For evaluation, we investigate the model fit and the performance of the model on unseen data. We observed a training $R^{2}$ between 0.85 and 0.98 , indicating that the model can capture a considerable proportion of the variance within the data. For unseen data, we split the benchmark data into a training and test set. We utilize a different number of training configurations to create a performance model. We investigate the root mean squared error of the predictions on the unseen test data of the remaining configurations. Figure 7 illustrates the results.

A small number of observations, i.e., 2-3 training configurations are enough to create a well-performing model. Due to the small amount of data, it can easily be used to identify optimal configurations for production systems. In general, the Lambda/Kinesis is more predictable than the Dask/Kafka model. For Dask, we observe a higher RSME for short-running tasks, i. e., smaller message and model sizes. For these configurations, the contention and coherence caused by the shared resources are higher, making the prediction is less precise.

\section{CONCLUSION AND FUture WORK}

We presented the Pilot-Streaming on serverless and StreamInsight designed to support the development, deploy- 

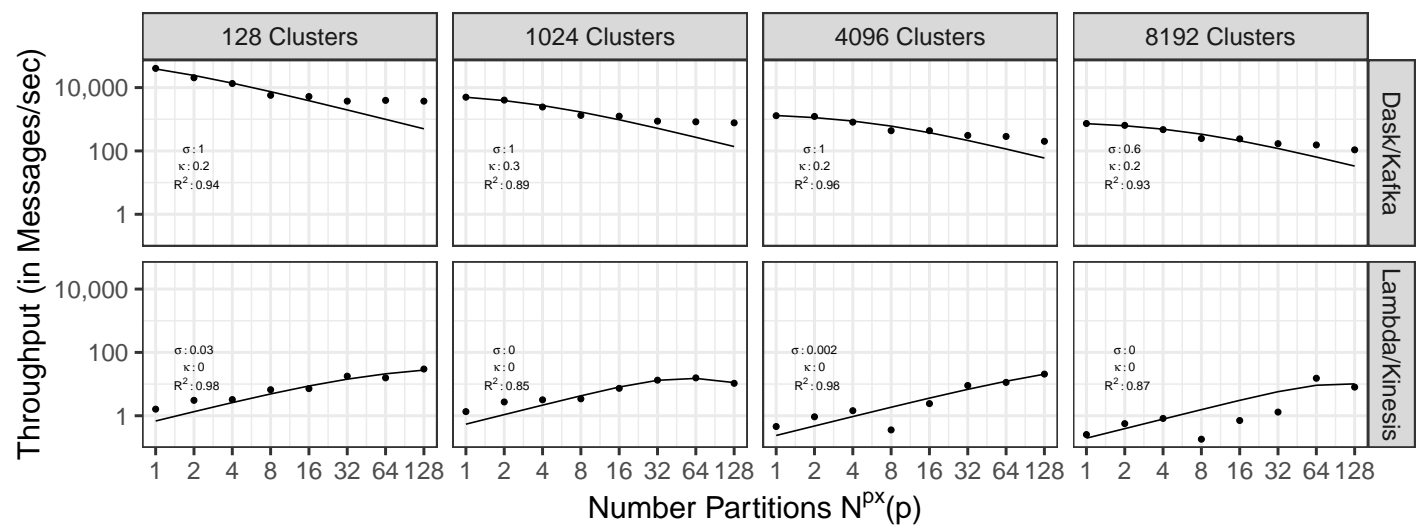

Fig. 6: Model Fit Universal Scalability Law applied to Lambda and Dask Streaming (Wrangler): The figure shows how the USL model was fitted to different scenarios. In all scenarios, the message size is 16,000 points. USL is suitable to describe the performance of streaming applications and enables StreamInsight to quantify overheads. For Kinesis/Lambda, USL produces a very small $\sigma$ and $\kappa$ explaining the optimal scalability. For Kafka/Dask, we observed larger coefficients explaining the severe performance degradation.

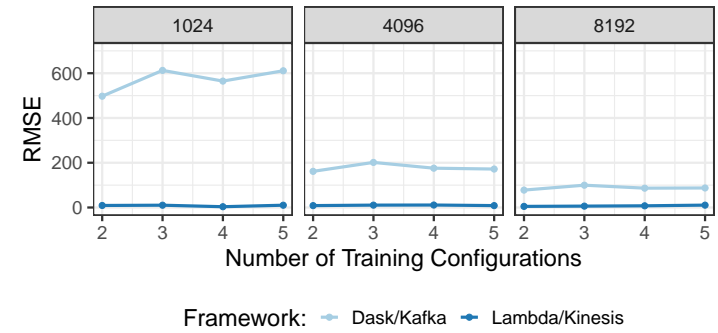

Fig. 7: RSME for Different Sizes of Training Data: A small number of observations is sufficient to obtain a well-fitted model.

ment, and execution of streaming applications. Pilot-Streaming on serverless demonstrated that the pilot abstraction's task model is well suited to express and manage serverless streaming workloads on serverless and HPC. The pilot abstraction enhances the expressiveness of serverless abstractions, e.g., by adding the ability to coordinate across multiple tasks required for many applications, such as machine learning. Further, Pilot-Streaming enables the interoperable use of serverless, cloud, and HPC.

StreamInsight provides comprehensive tools for creating performance experiments and analyze the data. We evaluated StreamInsight using different complex machine learning tasks and showed that the USL approach is well suited to predict the scaling properties of streaming applications requiring only small amounts of data. USL allows the quantification of properties, such as diminishing returns and communication bottlenecks. We found that using Kafka/Dask based stream processing on HPC provides better absolute performance than Kinesis/Lambda. However, the performance on HPC degrades when scaling due to I/O issues quickly. Kinesis/Lambda provide better scalability and predictability. Serverless is, however, subject to several limitations, e. g., the strict walltime of 15 minutes. Also, currently no GPUs are supported. Thus, for high-end workloads that require complex and large dependencies, e. g., TomoPy for light sources sciences or deep learning applications, serverless is not suitable.

We will extend StreamInsight to support advanced experimentation-in-the-loop-computing scenarios making use of complex edge, fog and cloud infrastructure. We will enhance Pilot-Streaming to support FaaS infrastructures, in particular on edge and fog environments. With Greengrass, AWS supports the execution of Lambda functions on the edge. By moving serverless functions to the edge and thus, closer to the data, further optimizations are possible. We will integrate StreamInsight into the resource management algorithm of Pilot-Streaming so as to support predictive scaling, viz., the ability to adapt the resource allocations and configurations to changes in the incoming data rate(s). This will also enable the determination of the amount of throttling of data sources to guarantee processing.

The above represent some advanced resource management capabilities that emerging EILC scenarios will require. PilotStreaming and StreamInsight provide dynamic resource management and a predictive resource allocation - two initial but instrumental building blocks towards these capabilities.

Acknowledgements: We acknowledge support from NSF DIBBS 1443054 and NSF CAREER OAC 1253644. XSEDE computational resources were made available via XRAC allocation TG-MCB090174.

\section{REFERENCES}

[1] Pete Beckman, Jack Dongarra, Nicola Ferrier, Geoffrey Fox, Terry Moore, Dan Reed, and Micah Beck. Harnessing the Computing Continuum for Programming Our World. http://dsc.soic.indiana.edu/ publications/paper_computing_continuum-2.pdf, 2019.

[2] Geoffrey Fox, Shantenu Jha, and Lavanya Ramakrishnan. Stream 2015 final report. http://streamingsystems.org/finalreport.pdf, 2015.

[3] Y. Du, M. Chowdhury, M. Rahman, K. Dey, A. Apon, A. Luckow, and L. B. Ngo. A distributed message delivery infrastructure for connected vehicle technology applications. IEEE Transactions on Intelligent Transportation Systems, 19(3):787-801, March 2018.

[4] André Luckow, George Chantzialexiou, and Shantenu Jha. Pilotstreaming: A stream processing framework for high-performance computing. Proceedings of 14th IEEE eScience, 2018.

[5] Brookhaven National Laboratory. National synchrotron light source ii. https://www.bnl.gov/ps/, 2017. 
[6] Ioana Baldini, Paul C. Castro, Kerry Shih-Ping Chang, Perry Cheng, Stephen Fink, Vatche Ishakian, Nick Mitchell, Vinod Muthusamy, Rodric Rabbah, Aleksander Slominski, and Philippe Suter. Serverless computing: Current trends and open problems. CoRR, abs/1706.03178, 2017.

[7] Eric Jonas, Johann Schleier-Smith, Vikram Sreekanti, Chia-Che Tsai, Anurag Khandelwal, Qifan Pu, Vaishaal Shankar, Joao Menezes Carreira, Karl Krauth, Neeraja Yadwadkar, Joseph Gonzalez, Raluca Ada Popa, Ion Stoica, and David A. Patterson. Cloud programming simplified: A berkeley view on serverless computing. Technical Report UCB/EECS-2019-3, EECS, University of California, Berkeley, 2019.

[8] Ioana Baldini, Perry Cheng, Stephen Fink, Nick Mitchell, Vinod Muthusamy, Rodric Rabbah, Philippe Suter, and Olivier Tardieu. The serverless trilemma: Function composition for serverless computing. In Proceedings of the 2017 ACM SIGPLAN International Symposium on New Ideas, New Paradigms, and Reflections on Programming and Software, Onward! 2017, pages 89-103, New York, NY, USA, 2017. ACM.

[9] Andre Luckow, Mark Santcroos, Andre Merzky, Ole Weidner, Pradeep Mantha, and Shantenu Jha. $\mathrm{P}^{*}$ : A model of pilot-abstractions. IEEE 8th International Conference on e-Science, pages 1-10, 2012.

[10] Neil J. Gunther. A simple capacity model of massively parallel transaction systems. In Int. CMG Conference, 1993.

[11] AWS. Kinesis. https://aws.amazon.com/kinesis/, 2017.

[12] AWS. Lambda: Run code without thinking about servers. https://aws. amazon.com/lambda/, 2019.

[13] J. Kreps, N. Narkhede, and J. Rao. Kafka: A distributed messaging system for log processing. In Proceedings of 6th International Workshop on Networking Meets Databases (NetDB), Athens, Greece, 2011.

[14] Dask Development Team. Dask: Library for dynamic task scheduling. http://dask.pydata.org, 2016.

[15] Geoffrey C. Fox, Vatche Ishakian, Vinod Muthusamy, and Aleksander Slominski. Status of serverless computing and function-as-aservice(faas) in industry and research. CoRR, abs/1708.08028, 2017.

[16] Eric Jonas, Shivaram Venkataraman, Ion Stoica, and Benjamin Recht Occupy the cloud: Distributed computing for the $99 \%$. CoRR, abs/1702.04024, 2017.

[17] Vaishaal Shankar, Karl Krauth, Qifan Pu, Eric Jonas, Shivaram Venkataraman, Ion Stoica, Benjamin Recht, and Jonathan Ragan-Kelley. numpywren: serverless linear algebra. CoRR, abs/1810.09679, 2018.

[18] Arvind Arasu, Mitch Cherniack, Eduardo Galvez, David Maier, Anurag S. Maskey, Esther Ryvkina, Michael Stonebraker, and Richard Tibbetts. Linear road: A stream data management benchmark. In Proceedings of the 30th International Conference on Very Large Data Bases, VLDB '04, pages 480-491. VLDB Endowment, 2004.

[19] S. Chintapalli, D. Dagit, B. Evans, R. Farivar, T. Graves, M. Holderbaugh, Z. Liu, K. Nusbaum, K. Patil, B. J. Peng, and P. Poulosky. Benchmarking streaming computation engines: Storm, flink and spark streaming. In 2016 IEEE International Parallel and Distributed Processing Symposium Workshops (IPDPSW), volume 00, pages 1789-1792, 2016.

[20] Geoffrey C. Fox, Shantenu Jha, Judy Qiu, Saliya Ekanazake, and Andre Luckow. Towards a Comprehensive Set of Big Data Benchmarks, 2015.

[21] S. R. Sukumar, M. A. Matheson, R. Kannan, and S. Lim. Mini-apps for high performance data analysis. In 2016 IEEE International Conference on Big Data (Big Data), pages 1483-1492, Dec 2016.

[22] Geoffrey Fox, James A. Glazier, Jcs Kadupitiya, Vikram Jadhao, Minje Kim, Judy Qiu, James Sluka, Endre Somogyi, Madhav Marathe, Abhijin Adiga, Jiangzhuo Chen, Oliver Beckstein, and Shantenu Jha. Learning everywhere: Pervasive machine learning for effective high-performance computation, 022019.

[23] Nathaniel Kremer-Herman, Benjamin Tovar, and Douglas Thain. A lightweight model for right-sizing master-worker applications. In Proceedings of the International Conference for High Performance Computing, Networking, Storage, and Analysis, SC '18, pages 39:1-39:13, Piscataway, NJ, USA, 2018. IEEE Press.

[24] Shivaram Venkataraman, Zongheng Yang, Michael Franklin, Benjamin Recht, and Ion Stoica. Ernest: Efficient performance prediction for largescale advanced analytics. In 13th USENIX Symposium on Networked Systems Design and Implementation (NSDI 16), pages 363-378, Santa Clara, CA, 2016. USENIX Association.

[25] Omid Alipourfard, Hongqiang Harry Liu, Jianshu Chen, Shivaram Venkataraman, Minlan Yu, and Ming Zhang. Cherrypick: Adaptively unearthing the best cloud configurations for big data analytics. In 14th USENIX Symposium on Networked Systems Design and Implementation (NSDI 17), pages 469-482, Boston, MA, 2017. USENIX Association.
[26] Faria Kalim, Thomas Cooper, Huijun Wu, Yao Li, Ning Wang, Neng Lu, Maosong Fu, Xiaoyao Qian, Hao Luo, Da Cheng, et al. Caladrius: A performance modelling service for distributed stream processing systems. Technical Report University of Illinois, 2019.

[27] Andre Luckow, Lukas Lacinski, and Shantenu Jha. SAGA BigJob: An Extensible and Interoperable Pilot-Job Abstraction for Distributed Applications and Systems. In The 10th IEEE/ACM International Symposium on Cluster, Cloud and Grid Computing, pages 135-144, 2010.

[28] Andre Luckow, Mark Santcroos, Ashley Zebrowski, and Shantenu Jha. Pilot-Data: An Abstraction for Distributed Data. Journal Parallel and Distributed Computing, October 2014.

[29] Matei Zaharia, Mosharaf Chowdhury, Michael J. Franklin, Scott Shenker, and Ion Stoica. Spark: Cluster computing with working sets. In Proceedings of 2nd USENIX Conference on Hot Topics in Cloud Computing, HotCloud'10, Berkeley, CA, USA, 2010. USENIX Association.

[30] Jeyhun Karimov, Tilmann Rabl, Asterios Katsifodimos, Roman Samarev, Henri Heiskanen, and Volker Markl. Benchmarking distributed stream processing engines. IEEE 34th Int. Conf. on Data Eng. (ICDE), 2018.

[31] Neil J. Gunther. Unification of amdahl's law, logp and other performance models for message-passing architectures. In International Conference on Parallel and Distributed Computing Systems, PDCS 2005, November 14-16, 2005, Phoenix, AZ, USA, pages 569-576, 2005.

[32] Gene M. Amdahl. Validity of the single processor approach to achieving large scale computing capabilities. In Proceedings of the April 18-20, 1967, Spring Joint Computer Conference, AFIPS '67 (Spring), pages 483-485, New York, NY, USA, 1967. ACM.

[33] Neil J. Gunther and Stefan Moeding. USL: Analyze System Scalability with the Universal Scalability Law. https://cran.r-project.org/web/ packages/usl/index.html, 2017.

[34] J. MacQueen. Some methods for classification and analysis of multivariate observations. In Proceedings of the Fifth Berkeley Symposium on Mathematical Statistics and Probability, Volume 1: Statistics, pages 281-297, Berkeley, Calif., 1967. University of California Press.

[35] Scikit-Learn Community. Mini batch kmeans. https://scikit-learn.org/ stable/modules/generated/sklearn.cluster.MiniBatchKMeans.html, 2019. 